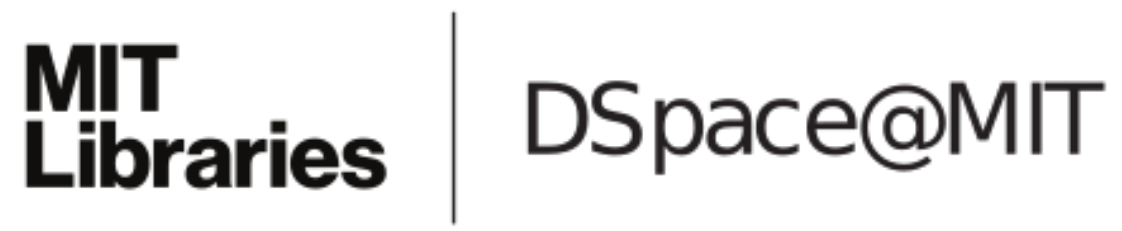

\author{
MIT Open Access Articles
}

Convex Models of Distribution System Reconfiguration

The MIT Faculty has made this article openly available. Please share how this access benefits you. Your story matters.

Citation: Taylor, Joshua A., and Franz S. Hover. "Convex Models of Distribution System Reconfiguration.” IEEE Trans. Power Syst. 27, no. 3 (August 2012): 1407-1413.

As Published: http://dx.doi.org/10.1109/tpwrs.2012.2184307

Publisher: Institute of Electrical and Electronics Engineers (IEEE)

Persistent URL: http://hdl.handle.net/1721.1/87726

Version: Original manuscript: author's manuscript prior to formal peer review

Terms of Use: Article is made available in accordance with the publisher's policy and may be subject to US copyright law. Please refer to the publisher's site for terms of use. 


\title{
Convex models of distribution system reconfiguration
}

\author{
Joshua A. Taylor, Member, IEEE, Franz S. Hover, Member, IEEE
}

\begin{abstract}
We derive new mixed-integer quadratic, quadratically constrained, and second-order cone programming models of distribution system reconfiguration, which are to date the first formulations of the AC problem that have convex continuous relaxations. Each model can be reliably and efficiently solved to optimality using standard commercial software. In the course of deriving each model, we obtain original quadratically constrained and second-order cone approximations to power flow in radial networks.
\end{abstract}

Index Terms-Distribution system reconfiguration, mixedinteger quadratic programming, second-order cone programming, load balancing

\section{INTRODUCTION}

D ISTRIBUTION system reconfiguration entails choosing the combination of open and closed switches that optimizes certain performance criteria while maintaining a radial network topology [1], [2]. Reconfiguration was originally addressed with branch exchange procedures [1], followed by genetic algorithms [3], simulated annealing [4], and other heuristics [5], to name a few. Such approaches are popular due to their broad applicability, e.g. for black-box models, but in turn may neglect intrinsic problem structure. Toward this end, in the present paper we develop new mixed-integer quadratic (QP) [6], quadratically constrained (QCP) [7], and second-order cone programming (SOCP) [8] formulations for reconfiguration.

When all variables are continuous, problems in these classes are always convex, and admit highly efficient and reliable polynomial-time algorithms [7]. As is well-known, each class becomes NP-hard when some of the variables are discrete. However, many integer programming algorithms, such as branch-and-bound, maintain excellent performance when efficiently computable bounds are available, and so it is highly desirable to have convex, polynomial-time continuous relaxations underlying mixed-integer problems; this is what implied by the use of the term convex in this work. In [9], a mixed-integer nonlinear programming formulation was solved using Benders decomposition [10]; in the same spirit, we solve mixed-integer, nonlinear - but convex - formulations. We summarize available algorithms for each framework in Sections III-A and III-C.

The contributions of this paper are two-fold. First, three new convex models are introduced, which we show in Section IV to be practical and competitive with existing methods,

J. Taylor and F. Hover are with the Department of Mechanical Engineering, Massachusetts Institute of Technology, Cambridge, MA, 02139 USA e-mail: jat1@mit.edu and hover@mit.edu.

This work was supported by the Office of Naval Research, Grant N0001402-1-0623, monitored by Dr. T. Ericsen. and two new approximate radial power flow formulations are obtained in their derivation. The second novel aspect of this work is that, to our knowledge, the models represent the first convex approximations to the $\mathrm{AC}$ version of the problem, a methodology which has seen past success and recent development in neighboring contexts such as optimal power flow [11], [12], transmission planning [13], [14], and unit commitment [15], [16]. Our formulations extend beyond reconfiguration to many radial network optimization problems, such as sizing and location of capacitors [17] and distributed generation [18].

A significant byproduct of our approach is convenience. The models may be solved using widely available, powerful commercial software, leaving to the user only programming the model while circumventing the algorithm. Although there are many tunable parameters within the standard software, default settings and automatic parameter selection usually lead to strong performance without user intervention. Similarly, starting points are accepted from the user, but are automatically generated if none are supplied.

\section{The DistFlow EQUATIONS}

We will make extensive use of the DistFlow equations for radial AC networks [1]. Let $p_{i j}$ and $q_{i j}$ denote the real and reactive powers at bus $i$ going to $j, v_{i}$ voltage magnitude, and $p_{i}^{L}$ and $q_{i}^{L}$ real and reactive loads at bus $i$. Since $v_{i}$ never appears in our formulation, we regard $v_{i}^{2}$ as a variable itself. Let $B$ be the set of buses, $W$ be the set of lines, and $r_{i j}$ and $x_{i j}$ be line resistance and reactance. Unless otherwise specified, single-subscript constraints are over all $i$ in $B$, and doublesubscripted constraints are over all $(i, j)$ in $W$. The DistFlow equations are given by

$$
\begin{gathered}
\sum_{k:(i, k) \in W} p_{i k}=p_{j i}-r_{i j} \frac{p_{j i}^{2}+q_{j i}^{2}}{v_{j}^{2}}-p_{i}^{L} \\
\sum_{k:(i, k) \in W} q_{i k}=q_{j i}-x_{i j} \frac{p_{j i}^{2}+q_{j i}^{2}}{v_{j}^{2}}-q_{i}^{L} \\
v_{i}^{2}=v_{j}^{2}-2\left(r_{i j} p_{j i}+x_{i j} q_{j i}\right)+\left(r_{i j}^{2}+x_{i j}^{2}\right) \frac{p_{j i}^{2}+q_{j i}^{2}}{v_{j}^{2}}
\end{gathered}
$$

\section{CONVEX MODELS}

\section{A. Quadratic programming}

In this section we use the simplified DistFlow equations [1], which are obtained by dropping all quadratic terms and (3). Let $W^{S}$ be the subset of $W$ with switches, $B^{F}$ be the subset of $B$ which are substations, $p_{i}^{F}$ and $q_{i}^{F}, i \in B^{F}$, be real and 
reactive powers from the substations, and $M$ be a sufficiently large disjunctive parameter. We obtain a mixed integer QP [6] for loss minimization by coupling the quadratic objective

$$
\min _{p, q, y, z} \sum_{(i, j)} r_{i j}\left(p_{i j}^{2}+q_{i j}^{2}\right)
$$

with the linear set of constraints

$$
\begin{aligned}
& \sum_{j:(i, j) \in W} p_{j i}-p_{i j}=p_{i}^{L} \quad i \in B / B^{F} \\
& \sum_{j:(i, j) \in W} q_{j i}-q_{i j}=q_{i}^{L} \quad i \in B / B^{F} \\
& \sum_{j:(i, j) \in W} p_{i j}=p_{i}^{F} \quad i \in B^{F} \\
& \sum_{j:(i, j) \in W} q_{i j}=q_{i}^{F} \quad i \in B^{F} \\
& 0 \leq p_{i j} \leq M z_{i j} \\
& 0 \leq q_{i j} \leq M z_{i j} \\
& z_{i j} \geq 0 \\
& z_{i f}=0 \quad f \in B^{F} \\
& z_{i j}+z_{j i}=1 \quad(i, j) \in W \backslash W^{S} \\
& z_{i j}+z_{j i}=y_{i j} \quad(i, j) \in W^{S} \\
& \sum_{j:(i, j) \in W} z_{j i}=1 \quad i \in B \backslash B^{F} \\
& y_{i j} \in\{0,1\} \quad(i, j) \in W^{S}
\end{aligned}
$$

We refer to (7)-(16) as $\Omega$. Two continuous variables $z_{i j}$ and $z_{j i}$ are associated with each line designating which direction, if any, flow may travel. With each switched line is also associated a single binary variable $y_{i j}$, which is zero if the switch is open and one if closed. These radiality constraints are very similar to those of [19] and [9]; however, this formulation is slightly more general in that not every line need be a candidate switch, potentially saving significant computation time. For the sake of thoroughness and to account for differences between this and prior radiality formulations, we now show that this scheme always results in a radial configuration. The below argument extends to all subsequent models in this paper.

Assume $y$ is fixed, and let $W^{y}=\left\{(i, j) \in W^{S}: y_{i j}=1\right\} \cup$ $W \backslash W^{S}$, i.e. the set of closed switches and non-switched lines. Consider a path through $W^{y}$ beginning at a substation, which we label bus zero. For any $(0, i) \in W^{y}, z_{0 i}=1$, and, by (13), (14), and (15), $z_{i j}=1$ for any $j \in B \backslash\{0\}$; we see by induction along the path that any $z$ on a path from the substation must be oriented such that it is zero going 'in' and one 'out', and therefore no two paths originating at a substation can meet (forming a loop if they are from the same substation). If a loop is formed which does not contain a substation, its buses are not receiving any flow, and (5) and (6) cannot be satisfied. We conclude that $z$ must describe an arborescence for each substation; to do so, $W^{y}$ must itself be composed of trees, and hence any feasible $y$ results in a radial configuration.

We remark that there are more continuous variables in the above formulation than necessary: only a single $z$ is needed for each line due to the equality constraints (13) and (14). However, to ease exposition and because the limiting factor is the number of discrete and not continuous variables, we have opted for the above, slightly less efficient presentation. Moreover, desirable modeling flexibility is built in, e.g. a line $(i, j)$ through which power can only flow in one direction may be accommodated by explicitly setting $z_{i j}=0$ or $z_{j i}=1$. Note that in a feasible configuration, each connected component must have exactly one substation.

Continuous QPs have polyhedral feasible sets, and thus their mixed-integer counterparts enjoy fast algorithms similar to those for mixed-integer linear programming, namely cutting planes coupled with branch-and-bound [20]. Furthermore, they can be solved with nearly the same efficiency [6], and thus the model QP represents a practical option for real-time reconfiguration.

\section{B. Quadratically constrained programming}

If we extend our framework to QCP [7], we enhance our modeling capability in three ways. First, line flow limits can be expressed $p_{i j}^{2}+q_{i j}^{2} \leq S_{i j}^{2}$, where $S$ denotes line capacity; these are new constraints. Second, recognizing that the equalities in (5) and (6) are identical to requiring that the flow into a bus be greater than the load plus the outgoing flow, losses may be approximated within flow conservation by replacing (5) and (6) with

$$
\begin{gathered}
\sum_{j:(i, j) \in W} p_{j i}-p_{i j}-r_{i j}\left(p_{j i}^{2}+q_{j i}^{2}\right) \geq p_{i}^{L} \\
\sum_{j:(i, j) \in W} q_{j i}-q_{i j}-x_{i j}\left(p_{j i}^{2}+q_{j i}^{2}\right) \geq q_{i}^{L}
\end{gathered}
$$

and leaving the remaining constraints of $\mathrm{QP}$ unchanged. With losses accounted for in flow conservation, the sum of the substation flows

$$
\sum_{i \in B^{F}} p_{i}^{F}
$$

may be equivalently (and more efficiently) used as a loss reduction objective instead of (4). Third, the load balancing formulation of [2] is accommodated by adding the constraint

$$
p_{i j}^{2}+q_{i j}^{2} \leq S_{i j}^{2} t
$$

and minimizing the objective $t$.

In practice, QCPs are usually solved using SOCP algorithms, and thus we defer discussing this aspect to the next section.

\section{Second-order cone programming}

SOCP [8] is a polynomial-time generalization of convex QCP, which is solved using highly efficient interior point algorithms [21]. We derive an SOCP approximation of the DistFlow equations in which only the last term in (3) is dropped, allowing voltage to vary away from one pu. The approximation is obtained by replacing (17) and (18) with the 
following constraints, the set of which we denote $\Phi$ :

$$
\begin{gathered}
\tilde{p}_{i}=-p_{i}^{L}+\sum_{j:(i, j) \in W} p_{j i}-p_{i j} \\
\tilde{q}_{i}=-q_{i}^{L}+\sum_{j:(i, j) \in W} q_{j i}-q_{i j} \\
\tilde{v}_{i}^{2} \leq v_{j}^{2}+M\left(1-z_{j i}\right) \\
\tilde{v}_{i}^{2} \geq v_{j}^{2}-M\left(1-z_{j i}\right) \\
r_{i j}\left(p_{j i}^{2}+q_{j i}^{2}\right) \leq \tilde{v}_{i}^{2} \tilde{p}_{i} \\
x_{i j}\left(p_{j i}^{2}+q_{j i}^{2}\right) \leq \tilde{v}_{i}^{2} \tilde{q}_{i} \\
v_{i}^{2} \leq v_{j}^{2}-2\left(r_{i j} p_{j i}+x_{i j} q_{j i}\right)+M\left(1-z_{j i}\right) \\
v_{i}^{2} \geq v_{j}^{2}-2\left(r_{i j} p_{j i}+x_{i j} q_{j i}\right)-M\left(1-z_{j i}\right) \\
v_{i}^{2}=1 \mathrm{pu} \quad i \in B^{F}
\end{gathered}
$$

We are again using disjunctive constraints in (23), (24), (27), and (28), which are only 'active' when $z_{j i}=1$. The purpose of the extra variables $\tilde{p}_{i}$ and $\tilde{q}_{i}$ is to put the constraints into an SOCP form recognizable by commercial solvers. Note that for a fixed network, these constraints and the quadratic constraints of the previous section constitute new radial power flow approximations.

As stated, continuous SOCPs can be solved very quickly using interior point algorithms. The mixed-integer counterpart has not attained the same maturity as mixed-integer linear or QP, but is handled by commercial solvers using branchand-bound routines. Furthermore, cutting planes and improved continuous relaxation procedures are active areas of research [22]-[24], and thus we can expect substantial improvements in the near future.

As we will see in the next section, the QCP and SOCP models can be more accurate than QP, but take considerably longer to solve using presently available algorithms. As the QP model does not account for losses, we expect that the improved accuracy seen by the QCP and SOCP models over the QP model grows with resistance and reactance.

\section{Relaxed optimality conditions}

The classical mixed-integer convex optimization algorithms the above models are tailored to are limited in that they will not terminate until a certifiably optimal solution is found; since all mixed-integer problems are NP-hard, this can be time consuming to the point that an uncertified or possibly suboptimal solution in less time is preferable [25]. Genetic algorithms and other heuristics do not share this problem, because optimality is not a termination criterion. However, there are many wellstudied ways of using classical mixed-integer programming algorithms heuristically by adjusting tolerances and thresholds, for example, by relaxing the integrality constraint, and only requiring that each discrete variable be within some small number of an integer. This is risky for reconfiguration because an infeasible rather than merely suboptimal solution could be returned. In the next section, we briefly examine modification to branch-and-bound in which, if $z$ is the objective of the best value found so far and $k$ is a small number, $z(1-k)$ is used instead to eliminate candidate solutions more rapidly [26].

\section{Computational EXAMPles}

In this section we use each model to reconfigure the 32bus test system of [1] and the 70-bus test system of [27] for the loss minimization and load balancing objectives as given in sections III-A and III-B, and maximizing minimum voltage. The latter is accommodated linearly by maximizing $t$ and adding the constraints

$$
t \leq v_{i}^{2} \text { for all } i .
$$

We note that minimum voltage maximization with the simplified DistFlow equations is actually a linear program. For load balancing, the p.u. capacity of each line was somewhat arbitrarily assumed to be the reciprocal of its impedance's magnitude. Table I below summarizes the objective and constraint formulations used in each example.

TABLE I

OBJECTIVES AND CONSTRAINTS FOR THE EXAMPLES

\begin{tabular}{c||c|c|c}
\hline Model & QP & QCP & SOCP \\
\hline \hline \multicolumn{4}{c}{ Loss minimization } \\
\hline Objective & 4 & 19 & 19 \\
Constraints & $\Omega, 5,6$ & $\Omega, 17,18$ & $\Omega, \Phi$ \\
\hline \hline \multicolumn{4}{c}{ Load balancing } \\
\hline Objective & - & $t$ & $t$ \\
Constraints & - & $\Omega, 17,18,20$ & $\Omega, \Phi, 20$ \\
\hline \hline \multicolumn{4}{c}{ Minimum voltage maximization } \\
\hline Objective & $t$ & $t$ & $t$ \\
Constraints & $\Omega, 5,6,27-30$ & $\Omega, 17,18,27-30$ & $\Omega, \Phi, 30$ \\
\hline
\end{tabular}

The resulting mixed-integer programs were solved using the modeling language AMPL [28] and solver CPLEX [29] on a desktop computer representative of current standards. Tables II and IV give per unit objectives and computation times for each model. Load flow solutions were computed using MATPOWER [30] for the configuration obtained by each model, and the corresponding objective value is shown in the second row of each block.

TABLE II

OPTIMIZATION OBJECTIVES, LOAD FLOW VALUES, AND COMPUTATION TIMES FOR THE 32-BUS TEST SYSTEM OF [1]

\begin{tabular}{c|lll}
\hline Model & QP & QCP & SOCP \\
\hline \hline \multicolumn{4}{|c}{ Loss minimization } \\
\hline Objective & 0.01273 & 0.01343 & 0.01404 \\
Load flow & 0.01395 & 0.01395 & 0.01395 \\
Time (s) & 0.21 & 1.43 & 12.80 \\
\hline \hline \multicolumn{4}{|c}{ Load balancing } \\
\hline Objective & - & 0.04134 & 0.04169 \\
Load flow & - & 0.04146 & 0.04146 \\
Time (s) & - & 2.31 & 18.06 \\
\hline \multicolumn{4}{c}{ Minimum voltage maximization } \\
\hline \hline Objective & 0.94253 & 0.94107 & 0.94082 \\
Load flow & 0.9412871 & 0.9412865 & 0.9412871 \\
Time (s) & 2.09 & 7.34 & 18.67 \\
\hline
\end{tabular}

On the 32-bus system, each model found the same lossminimizing switch configuration [9], which was determined to be optimal by enumeration in [31]. The QCP and SOCP models each found the same load balancing solution, with open switches $(8,21),(9,10),(14,15),(28,29)$, and $(31,32)$. As stated earlier, this load balancing formulation cannot be 
modeled within QP. The QP and SOCP models each found the same solutions for maximizing minimum voltage, with switches $(7,8),(9,10),(14,15),(28,29)$, and $(32,33)$ open. The QCP model found a very slightly worse solution with $(9,10)$ open instead of $(10,11)$.

There is a vast literature on reconfiguration algorithms. In 2006, implementations of some of the most successful algorithms were compared [31]; these are shown in Table III along with the results of the QP model and the recent works of [32] and [9]. Of the methods which found the optimal solution, only the value reported in [9] is faster than ours.

TABLE III

COMPARISON OF COMPUTATION TIMES (S) FROM A 2006 COMPARATIVE STUDY (FIRST SIX), TWO NEWER METHODS, AND THE QP MODEL FOR LOSS MINIMIZATION ON THE 32-BUS TEST SYSTEM [31].

\begin{tabular}{c|cc}
\hline Algorithm & time (s) & Optimal? \\
\hline \hline$[33]$ & 1.99 & yes \\
{$[34]$} & 0.87 & yes \\
{$[35]$} & 0.14 & no \\
{$[36]$} & 1.66 & no \\
{$[31]$} & 0.96 & yes \\
{$[32]$} & 7.2 & yes \\
{$[9]$} & 0.055 & yes \\
QP & 0.21 & yes \\
\hline
\end{tabular}

The second, larger example, on which no particular approach exhibited better accuracy across objectives, is included to demonstrate the scaling of each approach's computation time. Objectives, load flow values, and times are shown in Table IV, and Tables V, VI, and VII contain corresponding optimal configurations. Only switches that are open in at least one solution are shown, with open switches denoted 0 and closed 1. The SOCP model is too slow for a system this size; clearly new algorithms must be developed before it is practical. The QP model retains much of its efficiency, particularly for loss minimization, for which it required under a second. The QCP model again took times between the QP and SOCP models.

TABLE IV

OPTIMIZATION OBJECTIVES, LOAD FLOW VALUES, AND COMPUTATION TIMES FOR THE 70-BUS TEST SYSTEM OF [27]

\begin{tabular}{c|lll}
\hline Model & QP & QCP & SOCP \\
\hline \hline \multicolumn{4}{|c}{ Loss minimization } \\
\hline Objective & 0.02640 & 0.02852 & 0.03031 \\
Load flow & 0.03016 & 0.03018 & 0.03016 \\
Time (s) & 0.50 & 10.10 & 11310.48 \\
\hline \hline \multicolumn{4}{|c}{ Load balancing } \\
\hline Objective & - & 0.4394 & 0.4428 \\
Load flow & - & 0.44126 & 0.44132 \\
Time (s) & - & 226.24 & 1306.94 \\
\hline \multicolumn{4}{c}{ Minimum voltage maximization } \\
\hline Objective & 0.9223 & 0.9191 & 0.9186 \\
Load flow & 0.9247 & 0.9199 & 0.9199 \\
Time (s) & 40.59 & 737.10 & 4738.44 \\
\hline
\end{tabular}

Regarding the disparity between computation times across objectives: it must be recalled that these formulations are all NP-hard, and while some of the solvers employed are mature technologies, it is impossible to guarantee an efficient route to a solution. That said, the times obtained by the QP model are attractive, and suggest real-time applicability.
TABLE V

LOSS MINIMIZATION SOLUTIONS FOR THE 70-BUS TEST SYSTEM OF [27]. ONLY SWITCHES THAT ARE OPEN IN AT LEAST ONE SOLUTION SHOWN, WITH OPEN SWITCHES DENOTED 0 AND CLOSED 1.

\begin{tabular}{c|ccc}
\hline Line & QP & QCP & SOCP \\
\hline $9-15$ & 0 & 0 & 0 \\
$21-27$ & 0 & 0 & 0 \\
$28-29$ & 0 & 0 & 0 \\
$37-38$ & 0 & 1 & 0 \\
$40-44$ & 0 & 0 & 0 \\
$43-38$ & 1 & 0 & 1 \\
$49-50$ & 0 & 0 & 0 \\
$62-65$ & 0 & 0 & 0 \\
$67-15$ & 0 & 0 & 0 \\
\hline
\end{tabular}

TABLE VI

LOAD BALANCING SOLUTIONS FOR THE 70-BUS TEST SYSTEM OF [27]

\begin{tabular}{c|cc}
\hline Line & QCP & SOCP \\
\hline $8-9$ & 1 & 0 \\
$14-15$ & 0 & 1 \\
$21-27$ & 0 & 0 \\
$27-28$ & 0 & 1 \\
$29-64$ & 1 & 0 \\
$42-43$ & 1 & 0 \\
$43-38$ & 0 & 1 \\
$45-60$ & 0 & 0 \\
$48-49$ & 0 & 0 \\
$62-65$ & 0 & 1 \\
$65-66$ & 1 & 0 \\
$67-15$ & 0 & 0 \\
\hline
\end{tabular}

Lastly, as described in Section III-D, we relax the optimality requirement for loss minimization with the QP model by varying the parameter relobjdiff $=\mathrm{k}$ through the CPLEX options. Table VIII shows that as $r$ is increased, computation time shrinks at the expense of the optimality of the QP objective, and the corresponding load flow value deteriorates as well.

TABLE VII

VOLTAGE MAXIMIZATION SOLUTIONS FOR THE 70-BUS TEST SYSTEM OF [27]

\begin{tabular}{c|ccc}
\hline Line & QP & QCP & SOCP \\
\hline $9-15$ & 0 & 0 & 0 \\
$21-27$ & 0 & 0 & 0 \\
$28-29$ & 0 & 0 & 0 \\
$36-37$ & 1 & 1 & 0 \\
$37-38$ & 0 & 1 & 1 \\
$43-38$ & 1 & 0 & 1 \\
$49-50$ & 0 & 0 & 0 \\
$58-59$ & 0 & 1 & 1 \\
$59-60$ & 1 & 0 & 0 \\
$62-65$ & 0 & 0 & 0 \\
$67-15$ & 0 & 0 & 0 \\
\hline
\end{tabular}

TABLE VIII

COMPUTATION TIME AND ATTAINED OBJECTIVE UNDER A RELAXED OPTIMALITY REQUIREMENT (FOR THE QP MODEL ON THE 70-BUS SYSTEM)

\begin{tabular}{c|llll}
\hline$k$ & 0 & 0.005 & 0.01 & 0.05 \\
\hline \hline Objective & 0.02640 & 0.02648 & 0.02662 & 0.02725 \\
Load flow & 0.03016 & 0.03029 & 0.03056 & 0.03143 \\
Time (s) & 0.50 & 0.39 & 0.38 & 0.19 \\
\hline
\end{tabular}




\section{SUMMARY AND FUTURE WORK}

We have introduced three new convex, mixed-integer programming models for distribution system reconfiguration, implicit in which are new approximations to power flow in radial networks. To the authors' knowledge, these models are the first $\mathrm{AC}$ formulations that can utilize a powerful class of convex optimization algorithms. On two examples, the quadratic programming model is by far the most efficient, yet also obtains good solutions, evidencing its practicality for very large systems. The second-order cone model appears to be slightly more reliable in producing good solutions, but is rather expensive computationally; it is however reasonable to expect substantial improvements in mixed-integer second-order cone programming algorithms in the near future, in which case the second-order cone and quadratically constrained programming models will become more scalable options. An additional appealing aspect of each model is the convenience of using established commercial software, the scalability of which can be enhanced by relaxing optimality criteria; we remark, however, that although we recommend the use of such tools, any algorithm may be used to solve these models, and will still benefit from their convexity.

\section{REFERENCES}

[1] M. Baran and F. Wu, "Network reconfiguration in distribution systems for loss reduction and load balancing," Power Delivery, IEEE Transactions on, vol. 4, no. 2, pp. $1401-1407$, Apr. 1989.

[2] H.-D. Chiang and R. Jean-Jumeau, "Optimal network reconfigurations in distribution systems: I: A new formulation and a solution methodology," Power Delivery, IEEE Transactions on, vol. 5, no. 4, pp. 1902-1909, Oct. 1990.

[3] K. Nara, A. Shiose, M. Kitagawa, and T. Ishihara, "Implementation of genetic algorithm for distribution systems loss minimum reconfiguration," Power Systems, IEEE Transactions on, vol. 7, no. 3, pp. $1044-1051$, aug 1992.

[4] H.-C. Chang and C.-C. Kuo, "Network reconfiguration in distribution systems using simulated annealing," Electric Power Systems Research, vol. 29, no. 3, pp. 227 - 238, 1994. [Online]. Available: http://www.sciencedirect.com/science/article/pii/0378779694900183

[5] J.-P. Chiou, C.-F. Chang, and C.-T. Su, "Variable scaling hybrid differential evolution for solving network reconfiguration of distribution systems," Power Systems, IEEE Transactions on, vol. 20, no. 2, pp. 668 - 674, may 2005.

[6] D. Bienstock, "Computational study of a family of mixed-integer quadratic programming problems," Mathematical Programming, vol. 74, pp. 121-140, 1996.

[7] S. Boyd and L. Vandenberghe, Convex Optimization. New York, NY, USA: Cambridge University Press, 2004.

[8] M. S. Lobo, L. Vandenberghe, S. Boyd, and H. Lebret, "Applications of second-order cone programming," Linear Algebra and its Applications, vol. 284, pp. 193-228, Nov. 1998.

[9] H. Khodr, J. Martinez-Crespo, M. Matos, and J. Pereira, "Distribution systems reconfiguration based on OPF using Benders decomposition," Power Delivery, IEEE Transactions on, vol. 24, no. 4, pp. 2166 -2176, 2009.

[10] J. F. Benders, "Partitioning procedures for solving mixedvariables programming problems," Numerische Mathematik, vol. 4, pp. 238-252, 1962, 10.1007/BF01386316. [Online]. Available: http://dx.doi.org/10.1007/BF01386316

[11] J. Momoh, M. El-Hawary, and R. Adapa, "A review of selected optimal power flow literature to 1993. ii. newton, linear programming and interior point methods," Power Systems, IEEE Transactions on, vol. 14 no. 1, pp. $105-111$, Feb. 1999.

[12] J. Lavaei and S. H. Low, "Convexification of the optimal power flow problem," in Proc. Allerton Conf. on Communication, Control, and Computing, 2010
[13] R. Romero, A. Monticelli, A. Garcia, and S. Haffner, "Test systems and mathematical models for transmission network expansion planning," IEE Proceedings - Generation, Transmission and Distribution, vol. 149, no. 1 , pp. 27-36, 2002.

[14] J. A. Taylor and F. S. Hover, "Linear relaxations for transmission system planning," To appear, IEEE Trans. on Power Systems, 2011.

[15] T. S. Dillon, K. W. Edwin, H.-D. Kochs, and R. J. Taud, "Integer programming approach to the problem of optimal unit commitment with probabilistic reserve determination," Power Apparatus and Systems, IEEE Transactions on, vol. PAS-97, no. 6, pp. $2154-2166$, nov. 1978.

[16] M. Carrion and J. Arroyo, "A computationally efficient mixed-integer linear formulation for the thermal unit commitment problem," Power Systems, IEEE Transactions on, vol. 21, no. 3, pp. 1371 -1378, aug. 2006.

[17] M. Baran and F. Wu, "Optimal capacitor placement on radial distribution systems," Power Delivery, IEEE Transactions on, vol. 4, no. 1, pp. 725 -734 , Jan. 1989.

[18] G. Celli and F. Pilo, "Optimal distributed generation allocation in mv distribution networks," in Power Industry Computer Applications, 2001. PICA 2001. Innovative Computing for Power - Electric Energy Meets the Market. 22nd IEEE Power Engineering Society International Conference on, 2001.

[19] K. Butler, N. Sarma, and V. Ragendra Prasad, "Network reconfiguration for service restoration in shipboard power distribution systems," Power Systems, IEEE Transactions on, vol. 16, no. 4, pp. 653-661, Nov 2001.

[20] A. Schrijver, Theory of Linear and Integer Programming. John Wiley \& Sons, June 1998

[21] F. Alizadeh and D. Goldfarb, "Second-order cone programming," Mathematical Programming, vol. 95, pp. 3-51, 2003, 10.1007/s10107002-0339-5. [Online]. Available: http://dx.doi.org/10.1007/s10107-0020339-5

[22] J. P. Vielma, S. Ahmed, and G. L. Nemhauser, "A lifted linear programming branch-and-bound algorithm for mixed-integer conic quadratic programs," INFORMS J. on Computing, vol. 20, pp. 438-450, July 2008.

[23] S. Drewes, "Mixed integer second order cone programming," Ph.D. dissertation, Technischen Universität Darmstadt, 2009.

[24] A. Atamturk and V. Narayanan, "Conic mixed-integer rounding cuts," Mathematical Programming, vol. 122, pp. 1-20, 2010.

[25] L. A. Wolsey, Integer programming. Wiley-Interscience, 1998.

[26] D. P. Bertsekas, Nonlinear Programming. Athena Scientific, 2004.

[27] D. Das, "Reconfiguration of distribution system using fuzzy multiobjective approach," International Journal of Electrical Power and Energy Systems, vol. 28, no. 5, pp. 331 - 338, 2006.

[28] R. Fourer, D. M. Gay, and B. W. Kernighan, AMPL: A Modeling Language for Mathematical Programming. Duxbury Press, November 2002.

[29] "IBM ILOG CPLEX." [Online]. Available: http://www01.ibm.com/software/integration/optimization/cplex-optimizer/

[30] R. D. Zimmerman, C. E. Murillo-Sanchez, and R. J. Thomas, "MATPOWER: Steady-state operations, planning, and analysis tools for power systems research and education," Power Systems, IEEE Transactions on, vol. PP, no. 99 , pp. $1-8$, June 2010.

[31] F. Gomes, S. Carneiro, J. Pereira, M. Vinagre, P. Garcia, and L. R. de Araujo, "A new distribution system reconfiguration approach using optimum power flow and sensitivity analysis for loss reduction," Power Systems, IEEE Transactions on, vol. 21, no. 4, pp. $1616-1623$, nov. 2006.

[32] B. Enacheanu, B. Raison, R. Caire, O. Devaux, W. Bienia, and N. HadjSaid, "Radial network reconfiguration using genetic algorithm based on the matroid theory," Power Systems, IEEE Transactions on, vol. 23, no. 1, pp. $186-195$, Feb. 2008.

[33] T. McDermott, I. Drezga, and R. Broadwater, "A heuristic nonlinear constructive method for distribution system reconfiguration," Power Systems, IEEE Transactions on, vol. 14, no. 2, pp. 478 -483, may 1999.

[34] S. Goswami and S. Basu, "A new algorithm for the reconfiguration of distribution feeders for loss minimization," Power Delivery, IEEE Transactions on, vol. 7, no. 3, pp. 1484 -1491, jul 1992.

[35] D. Shirmohammadi and H. Hong, "Reconfiguration of electric distribution networks for resistive line losses reduction," Power Delivery, IEEE Transactions on, vol. 4, no. 2, pp. $1492-1498$, apr 1989.

[36] F. Gomes, J. Carneiro, S., J. Pereira, M. Vinagre, P. Garcia, and L. Araujo, "A new heuristic reconfiguration algorithm for large distribution systems," Power Systems, IEEE Transactions on, vol. 20, no. 3 , pp. $1373-1378$, aug. 2005. 